\title{
Cardiovascular Risk Factors and Evidence-Based Health Education
}

\author{
Marco Aurélio Lumertz Saffi ${ }^{1,2}$, Simoni Chiarelli da Silva Pokorski², \\ Eneida Rejane Rabelo-Silva ${ }^{2,3}$ \\ ${ }^{1}$ Graduate Program in Cardiology and Cardiovascular Sciences, Federal University of Rio Grande do Sul, Porto \\ Alegre, Brazil \\ ${ }^{2}$ Cardiovascular Division, Hospital de Clínicas de Porto Alegre, Porto Alegre, Brazil \\ ${ }^{3}$ School of Nursing, Federal University of Rio Grande do Sul, Porto Alegre, Brazil \\ Email: marco.saffi@gmail.com, simoni.chiarelli@gmail.com,
}

Received 10 January 2014; revised 16 February 2014; accepted 24 February 2014

Copyright @ 2014 by authors and Scientific Research Publishing Inc.

This work is licensed under the Creative Commons Attribution International License (CC BY).

http://creativecommons.org/licenses/by/4.0/

(c) (i) Open Access

\section{Abstract}

The number of deaths associated with cardiovascular events remains constant in many countries due to new therapeutic approaches for prevention and treatment of atherosclerosis. This condition is widely attributable to unhealthy outcomes in its association with risk factors such as smoking and sedentary behavior. Risk factors play a significant role in the progression of coronary artery disease. We conducted a review of the literature with the purpose of identifying primary risk factors for coronary disease, lifestyle change interventions, and expectations for a reduction in cardiovascular events on the basis of evidence-based health education strategies. In addition, we sought to contextualize this review so that nurses are trained to use these findings in their practice at different levels of care.

\section{Keywords}

Nursing; Coronary Disease; Risk Factors; Education; Nursing Research

\section{Introduction}

Cardiovascular disease (CVD) remains the leading cause of morbidity and mortality worldwide [1]. The primary cause of CVD is a combination of several risk factors (RFs) that correlate with the progression of endothelial injury and the development of atherosclerosis [2]. Over its course, the Framingham Heart Study has demon-

\footnotetext{
${ }^{*}$ Corresponding author.
} 
strated the importance of some of these factors in the development of cardiac and cerebrovascular disease. The main variables associated with atherosclerosis and its clinical manifestations were age, sex, systolic blood pressure, total cholesterol, smoking, and diabetes mellitus [3].

The INTERHEART multicenter study, a case-control study conducted in five continents including South America, sought to detect associations between a variety of RFs and acute myocardial infarction (AMI) in populations defined by ethnicity and/or geographic region. Nine RFs (smoking, dyslipidemia, hypertension, diabetes, obesity, diet, physical activity, alcohol intake, and psychosocial factors) were identified, and together contributed to over $90 \%$ of the AMI risk. The indicator associated with the greatest risk was the apolipoprotein B/apolipoprotein A1 (apoB/apoA1) ratio, followed by smoking; these factors were associated with a fourfold and threefold increases in risk respectively. Moderate alcohol consumption, daily consumption of fruits and vegetables, and regular physical activity were protective AMI [4].

The main objective of risk assessment and stratification programs is to identify the probability that an individual will develop atherosclerotic disease and its cardiovascular consequences. Within this context, in which RFs - whether alone or in combination - are shown to be directly related to cardiovascular events, we sought to identify precipitating RFs for coronary artery disease (CAD), lifestyle change interventions, perspective for cardiovascular event reduction, and education strategies [5]-[8].

This article will review contemporary data on the various aspects of the evidence-based health education process for management of RFs in patients with CVD—specifically, patients with CAD. Toward this end, the PubMed, BIREME, and MEDLINE electronic databases were searched for content published between the years 1990 and 2013, using the following search headings: nursing, coronary disease, risk factors, education, and nursing research.

\section{Cardiovascular Risk Factors and Their Evidence Base}

\subsection{Smoking}

Smoking remains a major public health issue, and a significant contributor to CVD-related morbidity and mortality [9]. In the pathophysiology of CAD, smoking contributes to endothelial dysfunction, inflammatory response, and changes in the lipid profile-all of which are integral elements in the progression of atherosclerosis. Tobacco causes a wide range of acute dynamic changes that increase the risk of cardiovascular events, including increases in heart rate and systemic vascular resistance and an imbalance in myocardial oxygen supply and demand [10]. Although the benefits of smoking cessation are evident [11], the most effective strategy to encourage patients to quit has yet to be established. All smokers should receive guidance on cessation from their health care providers. The use of interventions for modification of health-related behaviors is advocated in the search for smoking cessation [12]. A meta-analysis has demonstrated the efficacy of intensive behavioral interventions in encouraging smoking cessation, with odds ratios (ORs) of 1.49 (95\%CI 1.08 - 2.07) for individual counseling, 1.76 (95\%CI 1.11 - 2.93) for group counseling, and 1.58 (95\%CI 1.15 - 2.29) for telephone counseling; all were associated with superior rates of smoking cessation as compared with a control group [13]. Another meta-analysis found that nurse-led interventions were significantly more effective in encouraging smoking cessation (OR 1.43, 95\%CI 1.24 - 1.66) than a control group. These interventions consisted of individual counseling, including the use of explanatory handbooks [14].

\subsection{Sedentariness}

Physically active individuals have lower cardiovascular mortality rates [15]. There are clear association between physical activity and RFs for CAD; physical activity reduces blood pressure, improves glycemic control, prevents obesity, and improves the lipid profile and endothelial function [16]. Guidelines base their recommendations on four domains, which are key points for assessment of physical activity: 1) mode or type of activity; 2) frequency; 3) duration; and 4) intensity. Furthermore, it is recommended that adults avoid a sedentary lifestyle by engaging in physical activity for at least 150 minutes/week (moderate intensity) or 75 minutes/week (vigorous activity) for health benefits [17]. There is some evidence for these definitions. A study published in 2009 demonstrated a reduction in inflammatory marker levels, an increase in high-density lipoprotein (HDL) levels and an inverse association between moderate or vigorous physical activity (at least 20 minutes) and cardiovascular or other-cause mortality in patients with established CAD [18]. Regarding diabetes, a randomized study includ- 
ing 522 patients with glucose intolerance found an increase in HDL levels and reductions in triglycerides, body weight, and blood pressure in the intervention group (diet and exercise) as compared with controls at 3.2-year follow-up. The incidence of type 2 diabetes was $11 \%$ in the intervention group and $23 \%$ in the control group, which corresponds to a 58\% reduction in the incidence of diabetes in patients exposed to the lifestyle intervention, which included physical activity [19].

\subsection{Hypertension}

Arterial hypertension (HTN) is an independent risk factor for CVD, accounting for $25 \%$ of mortality in this setting. This risk increases in a progressive and linear fashion as blood pressure rises above 115/75 mmHg. In patients with hypertension, setting this blood pressure as a goal and reversing modifiable RFs leads to a reduction in cardiovascular morbidity and mortality [20]. Lifestyle change interventions should be recommended to all patients with hypertension. These interventions include weight loss to a target body mass index (BMI) of 18.5 $24.9 \mathrm{~kg} / \mathrm{m}^{2}$, daily fruit and vegetable consumption, reduction in saturated fat and cholesterol intake, smoking cessation, restriction of salt intake to $<2-4 \mathrm{~g} /$ day, restriction of alcohol intake to $10-30 \mathrm{~g} /$ day, and regular physical activity (150 minutes/week) [21].

\subsection{Dyslipidemia}

Elevated levels of low-density lipoprotein (LDL) and low levels of HDL are associated with atherogenesis, atherothrombosis, and clinical manifestations of cardiovascular disease [2]. The INTERHEART study showed that dyslipidemia was associated with myocardial infarction, with a 49\% population-attributable risk [4]. According to the $5^{\text {th }}$ Brazilian Guideline for Dyslipidemias and Prevention of Atherosclerosis (V Diretriz Brasileira de Dislipidemias e Prevenção da Aterosclerose), the LDL target for intermediate-risk patients should be $<100$ $\mathrm{mg} / \mathrm{dL}$. For high-risk patients (i.e., those with established CAD and multiple RFs), the LDL target is lowered to $70 \mathrm{mg} / \mathrm{dL}$. In patients on hypolipidemic agents, the recommended target is a 30\% - 40\% reduction in LDL from baseline, and for low-risk patients, the target is $\sim 160 \mathrm{mg} / \mathrm{dL}$. In addition, lifestyle changes are recommended for patients in all risk categories. Weight loss increases HDL by $5 \%-20 \%$, smoking cessation increases it by $5 \%$, and regular physical activity, by up to $30 \%$. Hypertriglyceridemia is also associated with increased cardiovascular risk, and is considered an independent RF for CAD [22].

\subsection{Diabetes}

Cardiovascular disease accounts for approximately $60 \%$ of all deaths of persons with diabetes. Individuals with type 1 or type 2 diabetes are exposed to a twofold to fourfold risk of cardiovascular events. Diabetes is also an independent RF for progression of atherosclerosis [23]. Abnormal blood sugar levels are associated with other cardiovascular RFs. Recent studies confirm the concept that care of patients with diabetes should be comprehensive and wide-ranging. Rather than simply treating hyperglycemia, RFs should also be addressed in an attempt to reduce the rate of cardiovascular events [24] [25].

\subsection{Obesity}

Obesity is associated with approximately $13 \%$ of cardiovascular deaths, and $80 \%$ of persons with CAD are overweight or obese [26]. A meta-analysis showed that weight loss brought on by diet and physical activity led to a decrease in total cholesterol and LDL, and increase in HDL, and improvement in blood pressure and diabetes management [27]. The evidence suggests that interventions should be longer, more intensive, and more frequent [25]. Obesity may be measured by parameters such as the BMI, the waist circumference (WC), and the waist-hip ratio (WHR) [4].

\subsection{Diet}

Adequate dietary counseling plays a role in the management of cardiovascular RFs and in the individualization of CAD treatment. Within this context, a systematic review sought to identify the best available evidence for dietary recommendations for patients with CVD. A reduction in saturated fat intake, replacement of saturated fats with unsaturated fats, and following a Mediterranean diet focused on fish, fruit, and vegetable intake had the 
greatest association with reduction in mortality and cardiovascular events [28]. The INTERHEART study demonstrated a 30\% reduction in AMI risk among individuals who consumed fruit and vegetables regularly [4]. A meta-analysis assessed the efficacy of a salt intake restriction intervention in hypertensive and normotensive subjects. A reduction in salt consumption to $6 \mathrm{~g}$ /day was associated with a 7.11-mmHg reduction in systolic blood pressure and a 3.88-mmHg reduction in diastolic blood pressure in hypertensive subjects, and a 3.57mmHg reduction in systolic and 1.66-mmHg reduction in diastolic blood pressure among normotensive subjects. These findings support evidence that a population-wide reduction in salt intake can reduce stroke-related mortality by $14 \%$ and cardiac mortality by $9 \%$ in hypertensive patients [29]. The Lifestyle Interventions for Blood Pressure Control (PREMIER) study, published in 2009, sought to assess the effect of two lifestyle interventions on reduction of the estimate of 10-year cardiovascular risk as compared with a control group. The main study findings were a $14 \%$ reduction in the Framingham risk score in patients who received guidance on cardiovascular RFs and a 12\% reduction in those who were also instructed to follow the Dietary Approaches to Stop Hypertension (DASH) diet, as compared with controls [6].

\subsection{Alcohol}

Several mechanisms have been proposed to explain the protective effect of moderate alcohol consumption in $\mathrm{CAD}$, as demonstrated by an increase in HDL levels and improvements in thrombolytic profile and platelet aggregation [30]. A meta-analysis of 28 cohort studies on the association between alcohol and CAD showed a risk reduction in patients who began to consume $<20 \mathrm{~g}$ /day of alcohol, with a relative risk (RR) of 0.80 (95\%CI 0.78 - 0.83), with evidence of a protective effect persisting at alcohol intake levels of up to $72 \mathrm{~g} /$ day (RR $=0.96$, 95\%CI 0.92 - 1.00) [31]. Although the INTERHEART study also showed a protective effect against AMI, with OR $=0.91$ (99\%CI 0.82 - 1.02) [4], there is no evidence from randomized controlled trials (RCTs) for a safe recommended level of daily alcohol intake. It bears stressing that alcohol consumption is also associated with social issues. Some individuals develop alcohol dependence, leading to digestive, renal, and cerebrovascular disease. Therefore, alcohol intake cannot be recommended as a cardiovascular prevention strategy [30].

\section{Cardiovascular Disease Prevention and Control Strategies}

Prediction of individual risk can play a useful role in clinical decision making, in determining the intensity of preventive interventions, and in guiding individualized management of RFs [7]. Within this setting, several multidisciplinary interventions for cardiovascular risk reduction have been established on the basis of evidence from RCTs of patients with CAD, including primary and secondary prevention strategies.

A systematic review highlighted CVD prevention guidelines that recommended lifestyle changes as identified by means of nine recommendations, namely: smoking cessation; regular physical activity; moderate alcohol consumption; adequate body weight; low saturated fat intake; fish, fruit, vegetable, legume, fiber, whole-grain, and nut consumption; and reduced salt intake. The estimates of risk reduction for mortality in patients with CAD were calculated as $35 \%$ for smoking cessation, $25 \%$ for physical activity, $20 \%$ for moderate alcohol consumption, and $45 \%$ for dietary changes [32].

Our research group recently published a RCT designed to assess the effect of systematic, individualized, nurse-led follow-up on a reduction in cardiovascular risk scores among patients with CAD. At 1-year follow-up, patients receiving the intervention had experienced a 1.7-point reduction $(-13.6 \%, P=0.011)$ in Framingham risk score. Significant improvements in secondary endpoints, such as weight, systolic blood pressure, and diastolic blood pressure were also observed in the intervention group [7]. Another RCT assessed nurse-led intervention strategies for patients with CAD over 1 to 4 years of follow-up. In the first year, patients in the intervention group experienced improvements in treatment adherence, blood pressure management, lipid profile, physical activity, and dietary control (all endpoints of interest except smoking cessation). At 4-year follow-up, there was additional or sustained improvement in all of these components, except for smoking and diet. The adjusted RR of all-cause mortality was 0.75 (95\%CI 0.58 - 0.98), and the RR for coronary artery events, 0.76 (95\%CI 0.58 1.0) [33]. However, many of these studies have been relatively short, and the intensity of the tested interventions is not feasible in a primary care setting in many countries. Furthermore, some clinical trials of health education-based interventions have failed to show significant reductions in cardiovascular morbidity and mortality [25]. 


\section{Health Education}

Lifestyle is a determinant of the overall health status of the population. To educate means to communicate, to establish communication channels and processes, and to provide points of reference. Culture and language are key elements of the educational process [34].

Educational policies should include a critical analysis that contrasts two models of health education, the "traditional" model and the "radical” model. The traditional model stresses a preventive approach toward health education, with an emphasis on the individual. This model assumes that health care providers know what constitutes a "healthy lifestyle", and does not take into account the influence of social determinants of disease. It employs a binary classification of healthy vs. unhealthy lifestyle, thus establishing what is "right” and "wrong” to exert a direct influence on individual behavior. Conversely, a new approach toward health education, based upon the tenets of radical model has been gaining ground in public health policies because it focuses on health promotion strategies that stimulates reflection on aspects of one's own reality, encouraging a search for and collective identification of the causes of said reality, examining it, and developing an action plan for change. The difference between the traditional and radical models is that the latter places greater emphasis on social change than on personal change. Within this context, nursing practice is challenged to find tools that can critically and effectively question the health issues imposed by a society undergoing the globalization process [35].

\section{Conclusions}

Evidence-based scientific knowledge is increasingly used to support the provision of health care in clinical practice. The various aspects of health education require direct associations between the available evidence, implementation of findings and assessment of outcomes and results obtained. In the setting of cardiovascular disease, nurses play an important role in the implementation of research findings, by integrating evidence-based knowledge into clinical decision making at the various levels of care. Patients with CAD benefit from cardiovascular risk reduction through the control of RFs such as hypertension, dyslipidemia, obesity, sedentary behavior, smoking, diabetes and alcohol intake, when counseled by nurses as to lifestyle modifications. Therefore, lifestyle counseling should be incorporated into clinical nursing practice regardless of setting.

Within this context, the present review article sought to provide inputs for the management of cardiovascular RFs in patients with CAD, by presenting relevant research findings that can support the establishment of intensive cardiovascular event reduction strategies and health education strategies in the search for better quality of life.

\section{Funding}

This work was supported by the Fundo de Incentivo à Pesquisa e Eventos (FIPE) do Hospital de Clínicas de Porto Alegre (HCPA).

\section{Conflict of Interest}

The authors declare that there is no conflict of interests regarding the publication of this article.

\section{References}

[1] The Top 10 Causes of Death. http://who.int/mediacentre/factsheets/fs310/en/

[2] Libby, P., Okamoto, Y., Rocha, V.Z., et al. (2010) Inflammation in Atherosclerosis: Transition from Theory to Practice. Circulation Journal, 74, 213-220. http://dx.doi.org/10.1253/circj.CJ-09-0706

[3] Fox, C.S., Evans, J.C., Larson, M.G., et al. (2004) Temporal Trends in Coronary Heart Disease Mortality and Sudden Cardiac Death from 1950 to 1999: The Framingham Heart Study. Circulation, 110, 522-527. http://dx.doi.org/10.1161/01.CIR.0000136993.34344.41

[4] Yusuf, S., Hawken, S., Ounpuu, S., et al. (2004) Effect of Potentially Modifiable Risk Factors Associated with Myocardial Infarction in 52 Countries (the INTERHEART Study): Case-Control Study. Lancet, 364, 937-952. http://dx.doi.org/10.1016/S0140-6736(04)17018-9

[5] Rabelo, E., Aliti, G., Domingues, F., et al. (2007) Enfermagem em Cardiologia Baseada em Evidência. Revista HCPA, 43-48. 
[6] Maruthur, N.M., Wang, N.Y. and Appel, L.J. (2009) Lifestyle Interventions Reduce Coronary Heart Disease Risk: Results from the PREMIER Trial. Circulation, 119, 2026-2031. http://dx.doi.org/10.1161/CIRCULATIONAHA.108.809491

[7] Saffi, M.A., Polanczyk, C.A. and Rabelo-Silva, E.R. (2013) Lifestyle Interventions Reduce Cardiovascular Risk in Patients with Coronary Artery Disease: A Randomized Clinical Trial. European Journal of Cardiovascular Nursing, 1-8.

[8] Dickson, V.V., Nocella, J., Yoon, H.W., et al. (2013) Cardiovascular Disease Self-Care Interventions. Nursing Research and Practice, $16 \mathrm{p}$.

[9] Gellert, C., Schöttker, B. and Brenner, H. (2012) Smoking and All-Cause Mortality in Older People: Systematic Review and Meta-Analysis. Archives of Internal Medicine, 172, 837-844. http://dx.doi.org/10.1001/archinternmed.2012.1397

[10] Ambrose, J.A. and Barua, R.S. (2004) The Pathophysiology of Cigarette Smoking and Cardiovascular Disease: An Update. Journal of the American College of Cardiology, 43, 1731-1737. http://dx.doi.org/10.1016/j.jacc.2003.12.047

[11] Wilson, K., Gibson, N., Willan, A., et al. (2000) Effect of Smoking Cessation on Mortality after Myocardial Infarction: Meta-Analysis of Cohort Studies. Archives of Internal Medicine, 160, 939-944. http://dx.doi.org/10.1001/archinte.160.7.939

[12] Force, U.S.P.S.T. (2009) Counseling and Interventions to Prevent Tobacco Use and Tobacco-Caused Disease in Adults and Pregnant Women: U.S. Preventive Services Task Force Reaffirmation Recommendation Statement. Annals of Internal Medicine, 150, 551-555. http://dx.doi.org/10.7326/0003-4819-150-8-200904210-00009

[13] Mottillo, S., Filion, K.B., Bélisle, P., et al. (2009) Behavioural Interventions for Smoking Cessation: A Meta-Analysis of Randomized Controlled Trials. European Heart Journal, 30, 718-730. http://dx.doi.org/10.1093/eurheartj/ehn552

[14] Rice, V.H. (1999) Nursing Intervention and Smoking Cessation: A Meta-Analysis. Heart Lung, 28, 438-454. http://dx.doi.org/10.1016/S0147-9563(99)70033-6

[15] Prasad, D.S. and Das, B.C. (2009) Physical Inactivity: A Cardiovascular Risk Factor. Indian Journal of Medical Sciences, 63, 33-42. http://dx.doi.org/10.4103/0019-5359.49082

[16] Taylor, R.S., Brown, A., Ebrahim, S., et al. (2004) Exercise-Based Rehabilitation for Patients with Coronary Heart Disease: Systematic Review and Meta-Analysis of Randomized Controlled Trials. American Journal of Medicine, 116, 682-692. http://dx.doi.org/10.1016/j.amjmed.2004.01.009

[17] Strath, S.J., Kaminsky, L.A., Ainsworth, B.E., et al. (2013) On Behalf of the American Heart Association Physical Activity Committee of the Council on Lifestyle and Cardiometabolic Health and Cardiovascular, C.rdiac Rehabilitation and Prevention Committee of the Council on Clinical Cardiology, and Council, Guide to the Assessment of Physical Activity: Clinical and Research Applications: A Scientific Statement from the American Heart Association, Circulation.

[18] Hamer, M. and Stamatakis, E. (2009) Physical Activity and Mortality in Men and Women with Diagnosed Cardiovascular Disease. The European Journal of Cardiovascular Prevention \& Rehabilitation, 16, 156-160. http://dx.doi.org/10.1097/HJR.0b013e32831f1b77

[19] Tuomilehto, J., Lindström, J., Eriksson, J.G., et al. (2001) Prevention of Type 2 Diabetes Mellitus by Changes in Lifestyle among Subjects with Impaired Glucose Tolerance. New England Journal of Medicine, 344, 1343-1350. http://dx.doi.org/10.1056/NEJM200105033441801

[20] Cardiologia, S.B.D., Hipertensão, S.B.D. and Nefrologia, S.B.D. (2010) VI Brazilian Guidelines on Hypertension. Arquivos Brasileiros de Cardiologia, 95, 1-51.

[21] Chobanian, A.V., Bakris, G.L., Black, H.R., et al. (2003) National Heart, and Blood Institute Joint National Committee on Prevention, Detection, E.aluation, and Treatment of High Blood Pressure, N.H.B.P.E.P.C. Committee, The Seventh Report of the Joint National Committee on Prevention, Detection, Evaluation, and Treatment of High Blood Pressure: The JNC 7 Report. The Journal of the American Medical Association, 289, 2560-2572. http://dx.doi.org/10.1001/jama.289.19.2560

[22] Xavier, H.T., Izar, M.C., Faria Neto, J.R., et al. (2013) V Diretriz Brasileira de Dislipidemias e Prevenção da Aterosclerose. Arquivos Brasileiros de Cardiologia, 1-22. http://dx.doi.org/10.5935/abc.2013S010

[23] Eberly, L.E., Cohen, J.D., Prineas, R., et al. (2003) Impact of Incident Diabetes and Incident Nonfatal Cardiovascular Disease on 18-Year Mortality: The Multiple Risk Factor Intervention trial Experience. Diabetes Care, 26, 848-854. http://dx.doi.org/10.2337/diacare.26.3.848

[24] Skyler, J.S., Bergenstal, R., Bonow, R.O., et al. (2009) Intensive Glycemic Control and the Prevention of Cardiovascular Events: Implications of the ACCORD, ADVANCE, and VA Diabetes Trials: A Position Statement of the American Diabetes Association and a Scientific Statement of the American College of Cardiology Foundation and the American Heart Association. Journal of the American College of Cardiology, 53, 298-304. http://dx.doi.org/10.1016/j.jacc.2008.10.008 
[25] Ebrahim, S., Beswick, A., Burke, M., et al. (2006) Multiple Risk Factor Interventions for Primary Prevention of Coronary Heart Disease. Cochrane Database of Systematic Reviews, CD001561.

[26] Flegal, K.M., Graubard, B.I., Williamson, D.F., et al. (2007) Cause-Specific Excess Deaths Associated with Underweight, Overweight, and Obesity. Journal of the American College of Cardiology, 298, 2028-2037. http://dx.doi.org/10.1001/jama.298.17.2028

[27] Avenell, A., Brown, T.J., McGee, M.A., et al. (2004) What Interventions Should We Add to Weight Reducing Diets in Adults with Obesity? A Systematic Review of Randomized Controlled Trials of Adding Drug Therapy, Exercise, Behaviour Therapy or Combinations of These Interventions. Journal of Human Nutrition and Dietetics, 17, $293-316$. http://dx.doi.org/10.1111/j.1365-277X.2004.00530.X

[28] Mead, A., Atkinson, G., Albin, D., et al. (2006) Dietetic Guidelines on Food and Nutrition in the Secondary Prevention of Cardiovascular Disease-Evidence from Systematic Reviews of Randomized Controlled Trials (Second Update, January 2006). Journal of Human Nutrition and Dietetics, 19, 401-419. http://dx.doi.org/10.1111/j.1365-277X.2006.00726.x

[29] He, F.J. and MacGregor, G.A. (2002) Effect of Modest Salt Reduction on Blood Pressure: A Meta-Analysis of Randomized Trials. Implications for Public Health. Journal of Human Hypertension, 16, 761-770. http://dx.doi.org/10.1038/sj.jhh.1001459

[30] Marmot, M.G. (2001) Alcohol and Coronary Heart Disease. International Journal of Epidemiology, 30, 724-729. http://dx.doi.org/10.1093/ije/30.4.724

[31] Corrao, G., Rubbiati, L., Bagnardi, V., et al. (2000) Alcohol and Coronary Heart Disease: A Meta-Analysis. Addiction, 95, 1505-1523. http://dx.doi.org/10.1046/j.1360-0443.2000.951015056.x

[32] Iestra, J.A., Kromhout, D., van der Schouw, Y.T., et al. (2005) Effect Size Estimates of Lifestyle and Dietary Changes on All-Cause Mortality in Coronary Artery Disease Patients: A Systematic Review. Circulation, 112, 924-934. http://dx.doi.org/10.1161/CIRCULATIONAHA.104.503995

[33] Murchie, P., Campbell, N.C., Ritchie, L.D., et al. (2003) Thain, Secondary Prevention Clinics for Coronary Heart Disease: Four Year Follow up of a Randomised Controlled Trial in Primary Care. British Medical Journal, 326, 84. http://dx.doi.org/10.1136/bmj.326.7380.84

[34] Silva, A.L. (2008) Nursing in the Era of Globalisation: Challenges for the 21st Century. Revista Latino-Americana de Enfermagem, 16, 787-790. http://dx.doi.org/10.1590/S0104-11692008000400021

[35] Oliveira, D.L. (2005) New Public Health and Health Promotion via Education: Between Tradition and Innovation. Revista Latino-Americana de Enfermagem, 13, 423-431. http://dx.doi.org/10.1590/S0104-11692005000300018 